\title{
Uniform Density in Lindenbaum Algebras
}

\author{
V. Yu. Shavrukov and Albert Visser
}

\begin{abstract}
In this paper we prove that the preordering $\lesssim$ of provable implication over any recursively enumerable theory $T$ containing a modicum of arithmetic is uniformly dense. This means that we can find a recursive extensional density function $F$ for $\lesssim$. A recursive function $F$ is a density function if it computes, for $A$ and $B$ with $A \lesssim B$, an element $C$ such that $A \lesssim C \lesssim B$. The function is extensional if it preserves $T$-provable equivalence. Secondly, we prove a general result that implies that, for extensions of elementary arithmetic, the ordering $\lesssim$ restricted to $\Sigma_{n}$-sentences is uniformly dense. In the last section we provide historical notes and background material.
\end{abstract}

\section{Introduction}

It is well known that the Lindenbaum algebras of theories that contain a modicum of arithmetic are dense with respect to the implication ordering. In this paper we will study a property that is stronger than density, to wit, uniform density. We prove that the Lindenbaum algebras of these theories are uniformly dense with respect to the implication ordering. We first provide the necessary definitions to formulate the result.

Consider any recursively enumerable theory $T$ that interprets the theory $\mathrm{R}$ introduced by Tarski, Mostowski, and Robinson in [21]. We define

$$
\begin{aligned}
& A \lesssim_{T} B \text { if and only if } T+A \vdash B, \\
& A \lesssim_{T} B \text { if and only if } A \lesssim_{T} B \text { and not } B \lesssim_{T} A, \\
& A \sim_{T} B \text { if and only if } A \lesssim_{T} B \text { and } B \lesssim_{T} A .
\end{aligned}
$$

Here $\lesssim_{T}$ is the "provable implication" ordering on $\mathfrak{L}_{T}$, the Lindenbaum sentence algebra of $T$. It is well known that $\lesssim_{T}$ is dense. We say that $\mathfrak{L}_{T}$ (or $\lesssim_{T}$ ) is uniformly dense if there is a recursive function $F$ such that

1. $F$ is a density function: that is, we have $A \lesssim_{T} F(A, B) \lesssim_{T} B$, whenever $A \lesssim_{T} B$, and if $A \sim_{T} B$, then $A \sim_{T} F(A, B) \sim_{T} B$; 
2. $F$ is extensional: if $A \sim_{T} A^{\prime}$ and $B \sim_{T} B^{\prime}$, then $F(A, B) \sim_{T} F\left(A^{\prime}, B^{\prime}\right)$.

We show that $\mathfrak{L}_{T}$ is uniformly dense for recursively enumerable theories $T$ that interpret R. Moreover, we can take the function $F$ to be elementary and, in some specific cases, even p-time computable.

We present our proof in Section 3. It consists of two stages. First we prove the desired result for Peano arithmetic PA or, more generally, for essentially reflexive theories (see Remark 1.1 for a discussion of essential reflexivity). Our construction delivers a p-time computable density function. This result is then generalized to all r.e. consistent theories that interpret $\mathrm{R}$ although the new density functions fall outside polynomial time-we don't know if this can be rectified.

A variant of the density question is obtained by imposing a restriction to a prescribed formula class. We explore this variant in Section 4. We prove a general result which implies, for example, that, for extensions $T$ of elementary arithmetic, the ordering $\lesssim_{T}$ restricted to $\Sigma_{n}$-sentences is uniformly dense.

The basic idea and the ingredients of our construction for PA come with a history. The sentences we produce are certain unique Rosser sentences of a kind studied by Smoryński [19], and they are the unique Gödel sentences of a certain Feferman predicate studied in Shavrukov [18]. Finally, they are Orey sentences. We will explain this background in Section 5. The reader who wants to just see the solution may, of course, skip Section 5.

Remark 1.1 A theory is essentially reflexive (uniformly essentially reflexive) if it proves reflection (resp., uniform reflection) for each of its finitely axiomatized subtheories. Here (uniform) reflection concerns a proof predicate that is formalized with respect to an interpretation $N$ of a weak arithmetic, like $\mathrm{S}_{2}^{1}$, in the given theory. Thus, a theory $U$ is essentially reflexive with respect to $N$ if it proves all principles of the form $\vdash \square_{U_{0}}^{N} A \rightarrow A$, where $A$ is a sentence of the language of $U$ and $U_{0}$ is any finitely axiomatized subtheory of $U$. A theory $U$ is uniformly essentially reflexive with respect to $N$ if it proves all principles of the form $\vdash \forall \vec{x}\left(\square_{U_{0}}^{N} A \vec{x} \rightarrow A \vec{x}\right)$, where $A \vec{x}$ is a formula of the language of $U$ with all variables among those shown and $U_{0}$ is any finitely axiomatized subtheory of $U$. We refer the reader to Beklemishev [1, Section 2] for a more extensive discussion.

Uniform essential reflexivity implies full induction with respect to the designated interpretation of the numbers. Conversely, a theory that satisfies full induction and is sequential is uniformly essentially reflexive (for the definition of sequential see Hájek and Pudlák [5] or Visser [26]). If we drop uniformity, essentially reflexive theories can be much weaker. For example, the minimal essentially reflexive extension of elementary arithmetic EA (here EA $=\mathrm{I} \Delta_{0}+$ Exp) is both a subtheory of PA and of EA plus all true $\Pi_{1}$-sentences. See also Visser [28].

Essentially reflexive theories form a natural class in the study of interpretability. For example, they satisfy the same propositional schemes for interpretability. See, for example, Visser [27] for a discussion.

\section{The Usual Proof of Density}

Our proof of uniform density for $\mathfrak{L}_{\mathrm{PA}}$ is a specific instance of the usual proof of the density of $\mathfrak{L}_{T}$, where $T$ is a recursively enumerable theory that interprets $\mathrm{R}$. We first present this usual proof. This proof will form a frame of reference for the rest of the paper. 


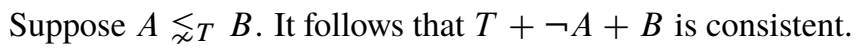

Let $C$ be any arithmetical sentence that is independent of $T+\neg A+B$; that is, $T+\neg A+B \nvdash C$ and $T+\neg A+B \nvdash \neg C$.

The essential ingredients of the proof that, for every consistent recursively enumerable theory $U$ that interprets $\mathrm{R}$, there exists a sentence $R$ that is independent of $U$ were provided by J. Barkley Rosser in his classical paper [16]. Here R is a very weak arithmetic introduced in [21].

We consider $D:=A \vee(C \wedge B)$. We claim that $A \lesssim_{\aleph_{T}} D \lesssim_{\aleph_{T}} B$.

First, we clearly have $A \lesssim_{T} D \lesssim_{T} B$.

Suppose $T+B \vdash A \vee(C \wedge B)$. By propositional logic, we find $T+\neg A+$ $B \vdash C$. Quod non.

Suppose $T+A \vee(C \wedge B) \vdash A$. By propositional logic, we have $T+\neg A+$ $B \vdash \neg C$. Quod non.

We can squeeze a little bit more information out of our construction. If $C$ is independent, then $\neg C$ is also independent. So we can construct $E:=A \vee(\neg C \wedge B)$.

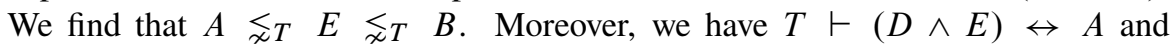
$T \vdash(D \vee E) \leftrightarrow B$. So we have two sentences $D$ and $E$ strictly between $A$ and $B$ such that $B$ is the supremum with respect to $\lesssim_{T}$ of $D$ and $E$ and $A$ is the infimum with respect to $\lesssim_{T}$ of $D$ and $E$.

Rosser's construction delivers a p-time mapping $A \mapsto C_{A}$, where $C_{A}$ is independent over $T+A$, provided that $T+A$ is consistent, for a given r.e. theory $T$ that interprets R. It is very unlikely, however, that the usual Rosser construction produces a uniform density function. For a brief discussion of this issue see Section 5.

In light of the proof given above, to prove p-time uniform density for PA, it is, modulo some simple details, sufficient to give a p-time construction $A \mapsto C_{A}$, where $C_{A}$ is independent over $\mathrm{PA}+A$, provided that $\mathrm{PA}+A$ is consistent and, if PA $\vdash A_{0} \leftrightarrow A_{1}$, then PA $\vdash C_{A_{0}} \leftrightarrow C_{A_{1}}$.

In the next section, we will provide a mapping $A \mapsto C_{A}$ that satisfies the desiderata.

\section{Uniform Density}

The first order of business for this section is the following.

Theorem 3.1 $\quad \mathfrak{L}_{\mathrm{PA}}$ is uniformly dense via a p-time computable density function. More generally, this result holds for all essentially reflexive sequential r.e. theories.

This theorem will later be extended to more theories.

We are interested in getting our density function as efficient as possible. In this case, we will construct a p-time computable function. To obtain an algorithm of the desired complexity, we will use efficiently coded syntax and base 2 numerals. See, for example, Buss [2, Chapter 7.3] or Hájek and Pudlák [5, Chapter V, Section 3].

We consider the sequence of theories $\mathrm{Ar}_{n}$, where $\mathrm{Ar}_{0}$ is $\mathrm{EA}$, also known as $\mathrm{I} \Delta_{0}+$ Exp, and $\operatorname{Ar}_{n+1}:=\mathrm{I} \Sigma_{n+1}$. These theories have the following important property.

Theorem 3.2 (EA proves that) for all $n, \mathrm{Ar}_{n+1}$ proves uniform $\Pi_{n+3}$-reflection for $\operatorname{Ar}_{n}$.

The theorem claims formalizability in EA of a result that was presumably first stated in Ono [12, Theorem 4.4]. That result is readily seen to be equivalent to I $\Sigma_{n+1}$ 
proving uniform $\Pi_{n+3}$-reflection for EA or even for pure predicate calculus, for $\mathrm{I} \Sigma_{n}+$ Exp can be axiomatized by a single $\Pi_{n+2}$-sentence (see [5, Theorems I.2.52 and V.5.6]). The latter equivalent coincides with the lemma in Leivant [7] modulo some ambiguity as to what Leivant's base theory $Z_{0}$ exactly is. The verification of EA-provability is best carried out along the proof of Tеорема 7 in [1]. We note that we have the following as an immediate consequence.

Corollary 3.3 (EA proves that) for any sentence $A$ in $\Sigma_{n+3}, \operatorname{Ar}_{n+1}+A$ proves uniform $\Pi_{n+3}$-reflection for $\operatorname{Ar}_{n}+A$.

We have the following definitions:

$\square_{A, x} B$ stands for $\operatorname{prov}_{\operatorname{Ar}_{x}+A}(\ulcorner B\urcorner)$,

$\diamond_{A, x} B$ stands for $\neg \operatorname{prov}_{\operatorname{Ar}_{x}+A}(\ulcorner\neg B\urcorner)$; that is, $\neg \square_{A, x} \neg B$.

As a first step towards the proof of Theorem 3.1 we need the formula $C_{A}$ :

$$
C_{A}:=A \wedge \forall x\left(\square_{A, x} \square_{A, x} \perp \rightarrow \square_{A, x} \perp\right) .
$$

We have the following useful lemma.

Lemma 3.4 Given any $n$, suppose $A \in \Sigma_{n+3}$. Then,

$$
\operatorname{Ar}_{n} \vdash C_{A} \leftrightarrow\left(A \wedge \forall x \geq n\left(\square_{A, x} \square \square_{A, x} \perp \rightarrow \square_{A, x} \perp\right)\right) .
$$

Proof By Corollary 3.3, $\operatorname{Ar}_{n}+A$ proves $\Sigma_{1}$-reflection for $\square_{A, k}$ with $k<n$. Hence,

$$
\operatorname{Ar}_{n}+A \vdash \forall x<n\left(\square_{A, x} \square \square_{A, x} \perp \rightarrow \square_{A, x} \perp\right) .
$$

The desired result is immediate.

In the next lemma, we show that $A \mapsto C_{A}$ is extensional.

Lemma 3.5 Suppose PA $\vdash A_{0} \leftrightarrow A_{1}$; then PA $\vdash C_{A_{0}} \leftrightarrow C_{A_{1}}$.

Proof Suppose PA $\vdash A_{0} \leftrightarrow A_{1}$. Then, for some $n, \operatorname{Ar}_{n} \vdash A_{0} \leftrightarrow A_{1}$. We can pick $n$ so large that $A_{0}, A_{1} \in \Sigma_{n+3}$. We have

$$
\begin{aligned}
\operatorname{Ar}_{n} \vdash C_{A_{0}} & \leftrightarrow A_{0} \wedge \forall x \geq n\left(\square_{A_{0}, x} \square_{A_{0}, x} \perp \rightarrow \square_{A_{0}, x} \perp\right) \\
& \leftrightarrow A_{1} \wedge \forall x \geq n\left(\square_{A_{1}, x} \square_{A_{1}, x} \perp \rightarrow \square_{A_{1}, x} \perp\right) \\
& \leftrightarrow C_{A_{1}} .
\end{aligned}
$$

Hence, PA $\vdash C_{A_{0}} \leftrightarrow C_{A_{1}}$.

Lemma 3.6 Suppose that PA $+A$ is consistent. Then $C_{A}$ is independent over $\mathrm{PA}+A$.

Proof Suppose that PA $+A \vdash C_{A}$. Then, for some $n, \operatorname{Ar}_{n}+A \vdash C_{A}$. We may assume that $A \in \Sigma_{n+3}$. It follows that $\operatorname{Ar}_{n}+A \vdash \square_{A, n} \square_{A, n} \perp \rightarrow \square_{A, n} \perp$. Hence, by Löb's theorem, $\operatorname{Ar}_{n}+A \vdash \square_{A, n} \perp$. We may conclude that PA $+A \vdash \perp$. Quod non.

Suppose that PA $+A \vdash \neg C_{A}$. Then, for some $n, \mathrm{Ar}_{n}+A \vdash \neg C_{A}$. We may assume that $A \in \Sigma_{n+3}$. We find, using Lemma 3.4,

$$
\operatorname{Ar}_{n}+A \vdash \exists x \geq n\left(\square_{A, x} \square_{A, x} \perp \wedge \diamond_{A, x} \top\right) .
$$

But then $\operatorname{Ar}_{n}+A \vdash \diamond_{A, n} \top$, contradicting the second incompleteness theorem. 
We put $F(A, B):=A \vee\left(C_{\neg A \wedge B} \wedge B\right)$. We note that this is defined for any arithmetical sentences $A$ and $B$. Moreover, we always have $A \lesssim_{\mathrm{PA}} F(A, B) \lesssim_{\mathrm{PA}} A \vee B$. By Lemma 3.5, $F$ is extensional. By Lemma 3.6, we have that, if $A \lesssim \mathrm{PA} B$, then

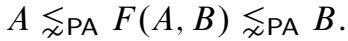

This concludes the proof of Theorem 3.1. We note that $F$ is p-time in $A$ and $B$.

Remark 3.7 We can put

$$
F_{0}(A, B):=A \vee\left(C_{\neg A \wedge B} \wedge B\right) \quad \text { and } \quad F_{1}(A, B):=A \vee\left(\neg C_{\neg A \wedge B} \wedge B\right),
$$

and then construct an infinite $\lesssim \mathrm{PA}$-antichain between $A$ and $B$ by considering

$$
\begin{aligned}
& D_{A, B, 0}:=F_{0}(A, B), \quad D_{A, B, 1}:=F_{0}\left(A, F_{1}(A, B)\right), \\
& D_{A, B, 2}:=F_{0}\left(A, F_{1}\left(A, F_{1}(A, B)\right)\right), \quad \ldots
\end{aligned}
$$

The mapping $H: A, B, n \mapsto D_{A, B, n}$ need not be p-time, but since this mapping is elementary we can represent it in PA. Since the $D_{A, B, n}$ have complexity (in the sense of the arithmetical hierarchy) bounded by the maximum of 2, the complexity of $A$ and that of $B$-say the complexity is $k(A, B)$-we can, using efficient numerals, replace $D_{A, B, n}$ by $E_{A, B, n}:=\operatorname{True}_{k(A, B)}(H(A, B, n))$. If we use a reasonable version of the definition of True ${ }_{k}$, the mapping $A, B, n \mapsto E_{A, B, n}$ becomes p-time. (Note that we do not need to worry about the length of the verifications of the usual properties of the True $_{k}$. We are only interested in the size of the formulas.)

Our proof can be immediately adapted to any essentially reflexive theory-like ZF: all the ingredients of the construction of $C$ are also present in such a theory.

Let $C_{A}^{\circ}:=\forall x\left(\square_{A, x} \square_{A, x} \perp \rightarrow \square_{A, x} \perp\right)$. Note that $C_{A}^{\circ}$ is $\Delta_{2}$ over I $\Sigma_{1}$ because it is I $\Sigma_{1}$-provably equivalent to $\diamond_{A} \top \vee \exists x\left(\square_{A, x+1} \perp \wedge \diamond_{A, x} \diamond_{A, x} \top\right)$.

Let us consider the relationship between $C_{A}^{\circ}$ and $\operatorname{con}(\mathrm{PA}+A)$.

Proposition 3.8 We have $\mathrm{PA}+\operatorname{con}(\mathrm{PA}+A) \vdash C_{A}^{\circ}$.

Proof Suppose that $A$ is $\Sigma_{n+3}$. We reason in PA + con $(\mathrm{PA}+A)$. Suppose that $\square_{A, x} \square_{A, x} \perp$. Then $\square_{A, \max \{x+1, n\}} \perp$ and, hence, $\square_{A} \perp$. Quod non. We may conclude $\neg \square_{A, x} \square_{A, x} \perp$, and, a fortiori, $C_{A}^{\circ}$.

Since, as we will show in Section $5, C_{A}^{\circ}$ is an Orey sentence of PA $+A$ and, provided that $\mathrm{PA}+A$ is consistent, $\operatorname{con}(\mathrm{PA}+A)$ is not an Orey sentence, $C_{A}$ is strictly between $A+\operatorname{con}(\mathrm{PA}+A)$ and $A$ over PA. In other words, $C_{A}^{\circ}$ is a reflection principle that is strictly between con $(\mathrm{PA}+A)$ and $\mathrm{T}$ over $\mathrm{PA}+A$.

Theorem 3.1 generalizes to theories containing $\mathrm{R}$ thanks to the following theorem.

Theorem 3.9 (Pour-El and Kripke [14, Theorem 2]) The Lindenbaum sentence algebras of all recursively enumerable, consistent theories that interpret $\mathrm{R}$ are effectively isomorphic.

For us, "effective isomorphism" means a recursive function from sentences of one theory to those of the other theory that, through provable equivalence, quotients down to an isomorphism between the two Lindenbaum algebras. The functions constructed in [14], however, possess further nice properties.

Pulling the density function of Theorem 3.1 off $\mathfrak{L}_{\mathrm{PA}}$ back to $\mathfrak{L}_{T}$ along an effective isomorphism $\mathfrak{L}_{T} \rightarrow \mathfrak{L}_{\mathrm{PA}}$, we obtain the following.

Corollary 3.10 The Lindenbaum sentence algebras of all recursively enumerable consistent theories that interpret $\mathrm{R}$ enjoy uniform density. 
It is seen from the proof of [14, Theorem 2] that the isomorphisms of Theorem 3.9 together with their inverses can be given by elementary functions (i.e., ones from Grzegorczyk class $\varepsilon^{3}$ ).

Accordingly, we are only able to claim elementarity rather than polynomial time for the second-hand density functions obtained via (the intended proof of ) Corollary 3.10. Our proof also forfeits the ability to have uniform density achieved by just mixing in an appropriate $\Delta_{2}$-sentence.

\section{Orderings of $\Sigma_{n}$-Sentences and Precomplete Lattices}

We address the question of uniform density for restricted classes of formulas in a somewhat more general setting.

An r.e. lattice $L$ is a pair of recursive functions $\vee$ and $\wedge$ defined on an r.e. subset field $L$ of $\omega$ together with an r.e. equivalence relation $\sim$ on field $L$ which is a congruence for $\vee$ and $\wedge$ and such that the quotient is a lattice. If that lattice is Boolean, then $L$ is called an r.e. Boolean algebra and, as is easily seen, has a recursive negation function. Lindenbaum sentence algebras of r.e. theories provide typical examples.

A density function for $L$ is a function $D:(\text { field } L)^{2} \rightarrow$ field $L$ such that, if $a \precsim b$, then $a \precsim D(a, b) \precsim b$, and $D(a, b) \sim a \sim b$ whenever $a \sim b$. The function $D$ is extensional (with respect to $\sim$ or $L$ ) if $\sim$ is a congruence for $D$. The lattice $L$ is uniformly dense if it admits an effective extensional density function-note that for $\mathfrak{L}_{T}$ this agrees with our earlier definition.

Montagna and Sorbi [11, Proposition 3.1(b)] extend Theorem 3.9 to all effectively inseparable (e.i.) r.e. Boolean algebras, that is, algebras where the -equivalence classes of (Boolean) 0 and 1 are effectively inseparable within field $L$. Hence Corollary 3.10 also holds for all e.i. r.e. Boolean algebras.

When the proof of Theorem 3.1 works for a theory $T$, it works equally well for the sublattice $\Sigma_{n} / T$ of $\mathfrak{R}_{T}$ determined by $\Sigma_{n}$-sentences provided that $n>1$ because $D(a, b)$ is a lattice polynomial in $a, b$, and a $\Delta_{2}$-sentence. In this section we handle $\Sigma_{1} / T$ using a different approach which starts with the definition of a precomplete numeration/equivalence.

A nontrivial equivalence relation $\sim$ on an r.e. subset field $\sim$ of $\omega$ is precomplete if to every partial recursive $f: \omega \rightarrow$ field $\sim$ there is a total recursive $F: \omega \rightarrow$ field $\sim$ that makes $f$ total modulo $\sim$, that is, $F(n) \sim f(n)$ whenever $f(n)$ converges. Reducing $f$ to a universal (field $\sim$ )-valued partial recursive function, we see that an index for $F$ can be found effectively in one for $f$.

An r.e. lattice $L$ is precomplete if its associated (r.e.) equivalence relation $\sim$ is (see [11, Section 2] or Selivanov [17, 4.4]). By $\precsim$ we denote the corresponding (r.e.) preorder on field $L$.

Example 4.1 (Visser $[23,1.6 .6]) \quad \Sigma_{n} / T$ is r.e. and precomplete whenever $T$ is a consistent r.e. extension of $\mathrm{EA}$.

Hint The mapping that assigns to $k$ the $\Sigma_{n}$-sentence $\exists y\left(y=f(\bar{k}) \wedge \operatorname{True}_{n}(y)\right)$ makes $f$ total modulo $T$-provable equivalence.

It is an open question whether $\Sigma_{1} / S_{2}^{1}$ or $\exists \Sigma_{1}^{\mathrm{b}} / S_{2}^{1}$ is precomplete (see [2] for definitions of $\exists \Sigma_{1}^{b}$ and $S_{2}^{1}$ ). 
Sentences of the form $\exists x T_{0}(\underline{n}, x)$, where $T_{0}$ is Kleene's T-predicate for the 0 -ary case, form an example of a class $\Gamma$, such that $\Gamma / S_{2}^{1}$ is r.e., precomplete, and is, modulo $\mathrm{S}_{2}^{1}$-provability, a sublattice of $\mathfrak{L}_{\mathrm{S}_{2}^{1}}$.

Mutual interpretability for finitely axiomatized sequential theories is also r.e. precomplete, since the interpretability ordering on finitely axiomatized sequential theories (modulo mutual interpretability) is p-time anti-isomorphic to $\Pi_{1} / \mathrm{EA}$. This uses the Friedman characterization of interpretability between finitely axiomatized theories (see, e.g., [25, Theorem 3.6]). Thus, the lattice of finitely axiomatized sequential interpretability degrees is (p-time) isomorphic to $\Sigma_{1} / E A$.

The r.e. extensions of PA in the language of PA modulo interpretability give us under an appropriate indexing an example of a precomplete numeration that is not recursively enumerable.

\section{Theorem 4.2 Any r.e. precomplete lattice is uniformly dense.}

Note that the theorem needs neither distributivity nor boundedness.

Here is the plan: given a recursive $F$, we are going to craft a partial recursive $f$. In other words, the construction below will effectively associate to an index $e$ for $F$ an index $c(e)$ for $f$. An index $t(c(e))$ for some $F^{\prime}$ making $f$ total modulo $\sim$ is then effective in $c(e)$. By the second recursion theorem there is an $e_{0}$ indexing the same function as $t\left(c\left(e_{0}\right)\right)$. For that $e_{0}$ we have $F^{\prime} \simeq F$. We may therefore assume from the outset that $F$ makes $f$ total modulo $\sim$.

Lastly, we put $D(a, b)=a \vee(F(a, b) \wedge b)$, which will be the desired extensional density function for $L$.

We fix effective enumerations $\left(\sim_{n}\right)_{n \in \omega}$ and $\left(\precsim_{n}\right)_{n \in \omega}$ of $\sim$ and $\precsim$, respectively, that satisfy the following:

for each $n \in \omega$, field $\sim_{n}=$ field $\precsim_{n}$ is a finite nonempty subset of field $L$;

$\sim_{n}$ is an equivalence relation;

$\sim_{n} \subseteq \sim_{n+1}$ and $\precsim_{n} \subseteq \precsim_{n+1}$;

$\sim=\bigcup_{n \in \omega} \sim_{n}$ and $\precsim=\bigcup_{n \in \omega} \precsim_{n}$.

Construction The construction of $f$ proceeds in stages. The following happens at Stage $n$.

(C1) Suppose that $a, b \in$ field $\sim_{n}$ and $f(a, b)$ has not yet been defined.

Let $a_{0}, b_{0} \in$ field $\sim_{n}$ be the minimal such that $a_{0} \sim_{n} a$ and $b_{0} \sim_{n} b$. Put $f(a, b)=F\left(a_{0}, b_{0}\right)$ unless $(a, b)=\left(a_{0}, b_{0}\right)$.

(C2) Suppose that $a \precsim_{n} b, f(a, b)$ has not yet been defined and $a \vee(F(a, b) \wedge$ b) $\precsim_{n} a$.

Put $f(a, b)=b$.

(C3) Suppose that $a \precsim_{n} b, f(a, b)$ has not yet been defined and $b \precsim_{n} a \vee$ $(F(a, b) \wedge b)$.

Put $f(a, b)=a$.

Claim 1 If $a \precsim b$, then $a \precsim D(a, b) \precsim b$. In particular, $a \sim b$ implies $a \sim D(a, b) \sim b$.

Proof This holds by virtue of the definition $D(a, b)=a \vee(F(a, b) \wedge b)$ regardless of the value of $F(a, b)$.

Claim $2 \quad f(a, b)$ is defined unless both $a$ and $b$ are minima of their respective $\sim$-equivalence classes. 
Proof Clause (C1) takes care of this.

Claim 3 If $f(a, b)$ is defined via clause (C2) or (C3), then $a \sim b$.

Proof Suppose that $f(a, b)$ is defined via clause (C2). We must then have $a \precsim b$, $a \vee(F(a, b) \wedge b) \precsim a$, and $F(a, b) \sim f(a, b)=b$, so $a \vee b \precsim a$; hence $a \sim b$.

Clause (C3) is treated similarly.

Claim $4 \quad D$ is extensional with respect to $\sim$.

Proof That the $\sim$-equivalence class of $D(a, b)$ only depends on those of $a$ and $b$ follows from Claim 1 for the case $a \sim b$. We may therefore assume $a \nsim b$. This implies, by Claim 3, that the only way to define $f\left(a^{\prime}, b^{\prime}\right)$ for $a^{\prime} \sim a$ and $b^{\prime} \sim b$ is via clause $(\mathrm{C} 1)$.

Assume that $a_{0}$ and $b_{0}$ are the minima of the $\sim$-equivalence classes of $a$ and $b$, respectively. We show by induction on $a+b$ that $F(a, b) \sim F\left(a_{0}, b_{0}\right)$ for all $a \sim a_{0}$ and $b \sim b_{0}$. Suppose $(a, b) \neq\left(a_{0}, b_{0}\right)$. By Claim 2, $f(a, b)$ is defined-via clause (C1). So $f(a, b)=F\left(a^{\prime}, b^{\prime}\right)$ where $a^{\prime} \sim a, b^{\prime} \sim b$, and $a^{\prime}+b^{\prime}<a+b$. Accordingly,

$$
F(a, b) \sim f(a, b)=F\left(a^{\prime}, b^{\prime}\right) \sim F\left(a_{0}, b_{0}\right)
$$

with the last equivalence holding by the induction hypothesis. Thus, we may conclude that $D(a, b) \sim D\left(a_{0}, b_{0}\right)$.

Claim 5 If $a \precsim b$, then $a \precsim D(a, b) \precsim b$.

Proof In view of Claim 1 it will suffice to exclude the situations $a \sim D(a, b)$ and $D(a, b) \sim b$.

Suppose $a \sim D(a, b)=a \vee(F(a, b) \wedge b)$. Let $a_{0}, b_{0}$ be the minima of $a$ 's and $b$ 's $\sim$-equivalence classes. Then $a_{0}, b_{0}$ also are minima of any $\sim_{n}$-equivalence classes they belong to. Thus clause (C1) cannot define $f\left(a_{0}, b_{0}\right)$. By Claim 3 neither can $(\mathrm{C} 2)$ nor $(\mathrm{C} 3)$. Yet clause $(\mathrm{C} 2)$ will sooner or later define $f\left(a_{0}, b_{0}\right)$ if nothing else does, a contradiction.

$D(a, b) \sim b$ is outruled in a similar fashion.

Claims 1, 4, and 5 amount to a proof of Theorem 4.2.

Corollary 4.3 For r.e. consistent $T$ extending EA the lattice $\Sigma_{n} / T$ is uniformly dense.

Remark 4.4 Using $\operatorname{True}_{n}(\cdots)$ as in Remark 3.7, one can bring down to p-time the complexity of any recursive function with values in $\Sigma_{n} / T$. The density functions for $\Sigma_{n} / T$ obtained through Theorem 4.2 however are already polynomial time because in $\Sigma_{n} / T$ totalization works by substitution (see the hint to Example 4.1) as does, for that matter, the second recursion theorem.

Corollary 4.5 The finitely axiomatized sequential theories are uniformly dense with respect to the interpretability preordering $\triangleleft$. The density function can be taken to be p-time.

Open Question 4.6 Are $\Sigma_{1} / \mathrm{S}_{2}^{1}$ and/or $\exists \Sigma_{1}^{\mathrm{b}} / \mathrm{S}_{2}^{1}$ uniformly dense?

Harvey Friedman shows in his Tarski lectures that the interpretability preordering on arbitrary finitely axiomatized theories of predicate logic has an effective density function. Is this ordering uniformly dense? 
Open Question 4.7 Do Lindenbaum sentence algebras admit uniform density functions that are monotone with respect to provable implication in (ideally) both arguments? What about precomplete r.e. lattices?

\section{Archaeology}

In this section we provide assorted background material that makes our construction of $C_{A}$ meaningful. We will sketch how the main ingredient of our formula $C_{A}$, to wit, the formula $C_{A}^{\circ}:=\forall x\left(\square_{A, x} \square_{A, x} \perp \rightarrow \square_{A, x} \perp\right)$, can be viewed as either a unique Rosser or Gödel fixed point. We first discuss the Rosser construction.

A standard way to produce independent sentences is the Rosser construction, invented by J. Barkley Rosser. The original paper is Rosser [16]. Rosser's construction has some extra good properties. The construction is verifiable in PA, and, after some careful inspection, even in EA. ${ }^{1}$ A second point is that Rosser's argument works for a very wide class of theories including the recursively enumerable extensions of the Tarski-Mostowski-Robinson theory R. Finally, the sentence produced by his construction, the Rosser sentence, is $\Sigma_{1}$ or $\Pi_{1}$, more specifically, $\exists \Pi_{1}^{\mathrm{b}}$ or $\forall \Sigma_{1}^{\mathrm{b}}$.

Can we use the original Rosser construction to obtain independent sentences in a uniform way? This does not look very promising: the sentences delivered by that construction are quite sensitive to implementation details. For example, suppose that we use a standard fixed point construction to obtain a Rosser sentence $R_{A}$ for $\mathrm{PA}+A$ and a Rosser sentence $R_{A^{\prime}}$ for PA $+A^{\prime}$. Suppose further that $A$ and $A^{\prime}$ are PA-provably equivalent. Then, $R_{A}$ and $R_{A^{\prime}}$ need not be PA-provably equivalent. The intensionality of the Rosser construction has, for example, been studied in Guaspari and Solovay [4] and Voorbraak [29]. However, several variants of the Rosser construction have been considered in the literature, and among these we find one that is sufficiently uniform. This Rosser construction was introduced by Craig Smoryński. As we will see, this Rosser construction can also be viewed as a Gödel construction.

Consider an r.e. extension $T$ of PA in the same language. Let $\tau:=\left(T_{n}\right)_{n \in \omega}$ be a recursive sequence of theories so that $\mathrm{I} \Sigma_{1}$ proves that

1. for all $n$ and $k$, if $n<k$, then $T_{n}$ is a subtheory of $T_{k}$;

2. the union of the $T_{n}$ is $T$;

3. for each $n, T_{n+1} \vdash \operatorname{con}\left(T_{n}\right)$.

We need the following definitions:

$\square_{\tau}^{\star} B$ stands for $\exists x \square_{T_{x}} B$; note that $\square_{\tau}^{\star} B$ is provably equivalent to $\square_{T} B$. Suppose that $C$ and $D$ are of the respective forms $\exists x C_{0}(x)$ and $\exists y D_{0}(y)$; then

$$
C<D:=\exists x\left(C_{0}(x) \wedge \forall y \leq x \neg D_{0}(y)\right)
$$

and

$$
C \leq D:=\exists x\left(C_{0}(x) \wedge \forall y<x \neg D_{0}(y)\right) .
$$

Note that $\square_{\tau}^{\star} B<\square_{\tau}^{\star} C$ is $I \Sigma_{1}$-provably equivalent to $\square_{T} B \wedge \neg\left(\square_{\tau}^{\star} C \leq \square_{\tau}^{\star} B\right)$. Thus, the formula $\square_{\tau}^{\star} B<\square^{\star} C$ is $\Delta_{2}$ over $\mathrm{I} \Sigma_{1}$. The case is similar for $\square_{\tau}^{\star} B \leq \square^{\star} C$.

The formula $\square_{\tau}^{\star} B<\square_{\tau}^{\star} C$ is equivalent over PA to $\exists x\left(\square_{T_{x}} B \wedge \neg \square_{T_{x}} C\right)$. It follows that the formula $\square_{\tau}^{\star} B<\square_{\tau}^{\star} \neg B$ is equivalent to $\square_{\tau}^{\star} B<\square_{\tau}^{\star} \perp$ which coincides 
with the Feferman predicate for $\tau$ defined as

$$
\triangle_{\tau} A:=\square_{\tau}^{\star} A<\square_{\tau}^{\star} \perp \text {. }
$$

We note that, over $\mathrm{EA}, \triangle_{\tau} A$ is equivalent to $\exists x\left(\square_{T_{x}} A \wedge \diamond_{T_{x}}^{\top}\right)$.

The Feferman predicate was introduced by Solomon Feferman in his classical paper [3]. The Feferman predicate is a sort of self-correcting provability predicate. It is related to trial and error predicates as studied in Putnam [15] and Jeroslow [6]. Feferman's aim in introducing it was not just the study of ways to escape the second incompleteness theorem but also applications to the study of relative interpretability.

Here is the central insight. We write

- $\nabla_{\tau} A$ for $\neg \triangle_{\tau} \neg A$;

- $U \triangleright V$ for: there is a relative interpretation of $V$ in $U$.

See, for example, [21], [3], Lindström [8] and Visser [25] for basic definitions concerning interpretations.

Theorem 5.1 We have $\left(T+\nabla_{\tau} A\right) \triangleright(T+A)$.

See [3] for the main ingredients of the proof. The basic idea of the result is that $\nabla_{\tau} A$ is a consistency statement of $T+A$. We can use the Henkin construction to build the desired interpretation.

We now consider the specialized sequences $v_{A}:=\left(\operatorname{Ar}_{n}+A\right)_{n \in \omega}$ for the theories $\mathrm{PA}+A$. We simply write $\square_{A}^{\star}$ for $\square_{v_{A}}^{\star}$, and so on.

By the Gödel fixed point lemma, we can find a sentence $R_{A}$, such that

$$
\mathrm{PA} \vdash R_{A} \leftrightarrow \neg\left(\square_{A}^{\star} R_{A}<\square_{A}^{\star} \neg R_{A}\right) .
$$

Thus $R_{A}$ is a Rosser sentence for the $\square_{A}^{\star}$. By our previous remarks, the sentence $R_{A}$ is also a Gödel sentence for the Feferman predicate $\triangle_{A}$, that is:

$$
\mathrm{PA} \vdash R_{A} \leftrightarrow \neg \triangle_{A} R_{A} .
$$

Smoryński investigates $R_{A}$ in his paper [19]. That paper was inspired by a study of a variant of the Rosser construction in the context of set theory by Kenneth McAloon [9].

Theorem 2.1 in [19] implies that $R_{A}$ is, up to provable equivalence, unique over $\mathrm{PA}+A$. By a minor addition to Smoryński's argument, one can show that the mapping $A \mapsto\left(A \wedge R_{A}\right)$ preserves PA-provable equivalence. Shavrukov [18] shows that uniqueness can fail under a choice of stratification sequence different from $\left(\operatorname{Ar}_{n}\right)_{n \in \omega}$.

Smoryński also shows that $R_{A}$ is independent over $\mathrm{PA}+A$, provided that $\mathrm{PA}+A$ is consistent. As we will see, Smoryński's Rosser sentence $R_{A}$ is (PA $+A$ )-provably equivalent to the sentence $\forall x\left(\square_{A, x} \square_{A, x} \perp \rightarrow \square_{A, x} \perp\right)$. So the independence of $R_{A}$ also follows from our Lemma 3.6.

Since the Feferman-Smoryński predicate explicates a notion of provability, it can be studied modally. This study was taken up in Montagna [10], Visser [24], and Shavrukov [18]. The latter paper studies the Feferman predicate over PA based on the sequence $\mathrm{Ar}_{n}$ with conclusions translatable to the hierarchy $\mathrm{Ar}_{n}+A$. Thus [18] contains an alternative, modal, proof of the uniqueness of $R_{A}$.

Recall that $C_{A}^{\circ}:=\forall x\left(\square_{A, x} \square_{A, x} \perp \rightarrow \square_{A, x} \perp\right)$. We show that $C_{A}^{\circ}$ is a Gödel sentence for $\triangle_{A}$.

Theorem 5.2 ([18, Exercise 2.7]) $\quad C_{A}^{\circ}$ is a Gödel sentence of $\triangle_{A}$ over PA $+A$. 
Proof We have, using Löb's theorem in the third step,

$$
\begin{aligned}
\mathrm{PA}+A \vdash \triangle_{A} C_{A}^{\circ} & \rightarrow \exists x\left(\square_{A, x} \forall y\left(\square_{A, y} \square_{A, y} \perp \rightarrow \square_{A, y} \perp\right) \wedge \diamond_{A, x} \top\right) \\
& \rightarrow \exists x\left(\square_{A, x}\left(\square_{A, x} \square_{A, x} \perp \rightarrow \square_{A, x} \perp\right) \wedge \diamond_{A, x} \top\right) \\
& \rightarrow \exists x\left(\square_{A, x} \square_{A, x} \perp \wedge \diamond_{A, x} \top\right) \\
& \rightarrow \neg C_{A}^{\circ} .
\end{aligned}
$$

We treat the other direction. Suppose $A \in \Sigma_{n+3}$. We work in PA $+A$. Suppose $\neg C_{A}^{\circ}$, that is,

$$
\exists x\left(\square_{A, x} \square_{A, x} \perp \wedge \diamond_{A, x} \top\right) .
$$

Clearly, it follows that $\exists x\left(\square_{A, x} \forall y \geq x \square_{A, y} \perp \wedge \diamond_{A, x} \top\right)$, and hence:

$$
\exists x\left(\square_{A, x} \forall y \geq x\left(\square_{A, y} \square_{A, y} \perp \rightarrow \square_{A, y} \perp\right) \wedge \diamond_{A, x} \top\right) .
$$

We note that we may assume that $x \geq n$, since for any standardly finite $k$, $\square_{A, k} \square_{A, k} \perp$ implies $\perp$. Hence, by the fact that $\operatorname{Ar}_{x}+A$ proves $\Sigma_{1}$-reflection for $\operatorname{Ar}_{y}+A$, for $y<x$, we find

$$
\exists x\left(\square_{A, x} \forall y<x\left(\square_{A, y} \square_{A, y} \perp \rightarrow \square_{A, y} \perp\right) \wedge \diamond_{A, x} \top\right) .
$$

Combining (i) and (ii), we find

$$
\exists x\left(\square_{A, x} \forall y\left(\square_{A, y} \square, A, y \rightarrow \square_{A, y} \perp\right) \wedge \diamond_{A, x} \top\right) .
$$

Of course (iii) is $\triangle_{A} C_{A}^{\circ}$.

Thus, we have shown that PA $+A \vdash C_{A}^{\circ} \leftrightarrow \neg \triangle_{A} C_{A}^{\circ}$.

So, $C_{A}^{\circ}$ is modulo PA-provable equivalence Smoryński's Rosser sentence for PA $+A$.

Open Question 5.3 Our proof of the extensionality of $A \mapsto C_{A}$ as well as that of unprovability of $C_{A}$ go through, with minor modifications, for any stratification sequence $\tau$ for PA satisfying our conditions. The consistency of $C_{A}^{\circ}$ with PA $+A$ is the only element of Theorem 3.1 that ostensibly depends on $\tau=\left(\operatorname{Ar}_{n}\right)_{n \in \omega}$ (or, more generally, on the "fast-growing" property of $\tau$ that each level proves enough reflection for the previous ones).

This makes us wonder if there exists a consistent theory of the form PA $+A$ together with a stratification sequence $\tau=\left(T_{n}\right)_{n \in \omega}$ such that PA $+A$ refutes $\forall x\left(\square_{A, T_{x}} \square_{A, T_{x}} \perp \rightarrow \square_{A, T_{x}} \perp\right)$.

A similar question can be asked of Theorem 5.2.

We end this section by showing that $C_{A}^{\circ}$ is an Orey sentence of PA $+A$.

Consider any theory $T$. A sentence $O$ in the language of $T$ is an Orey sentence of $T$ if $T \triangleright(T+O)$ and $T \triangleright(T+\neg O)$. Note that the negation of an Orey sentence is an Orey sentence. An Orey sentence $O$ of $T$ is clearly independent of $T$. Neither an Orey sentence nor its negation add interpretability strength to the given theory.

The idea of Orey sentences was introduced by Orey [13], who also provided the first known Orey sentence for PA. There are many salient natural Orey sentences. Two well-known examples are the parallel axiom over a suitable version of neutral geometry and the continuum hypothesis over ZFC. For essentially reflexive sequential theories, the Gödel sentence of a Feferman predicate for the theory is an Orey sentence-see below.

We will show that $C_{A}^{\circ}$ is an Orey sentence of PA $+A$. This sentence is still metamathematical and does involve coding, but it is, at least, self-reference-free. Since 
$C_{A}^{\circ}$ is a Gödel sentence of $\triangle_{A}$, the desired insight is immediate by the following theorem. (This theorem was also proved in [24].)

Theorem 5.4 Consider a consistent theory $T$ given by a sequence $\tau$ satisfying the conditions given above. Then any Gödel sentence of $\triangle_{\tau}$ is an Orey sentence for $T$.

Proof Let $G$ be a Gödel sentence of $\triangle_{\tau}$. We have

$$
\begin{aligned}
T+G & \vdash T+\nabla_{\tau} \neg G \\
& \triangleright T+\neg G, \\
T+\neg G & \vdash T+\triangle_{\tau} G \\
& \vdash T+\nabla_{\tau} G \\
& \triangleright T+G .
\end{aligned}
$$

In the second step of the second proof, we use $T \vdash \triangle_{\tau} \neg A \rightarrow \neg \triangle_{\tau} A$. Thus, we have both $(T+G) \triangleright(T+G)$, by the identity interpretation, and $(T+\neg G) \triangleright$ $(T+G)$. So, using a disjunctive interpretation, we find $T \triangleright(T+G)$. Similarly, $T \triangleright(T+\neg G)$.

\section{Note}

1. A modified argument even works in $\mathrm{I} \Delta_{0}+\Omega_{1}$. The basic idea of this argument is due to Švejdar [20]. For the verification that Švejdar's assumptions are fulfilled, see Verbrugge and Visser [22].

\section{References}

[1] Beklemishev, L. D., "Reflection schemes and provability algebras in formal arithmetic" (in Russian), Uspekhi Matematicheskikh Nauk, vol. 60, no. 2 (2005), pp. 3-78; English translation in Russian Mathematical Surveys, vol. 60 (2005), pp. 197-268. Zbl 1097.03054. MR 2152943. DOI 10.1070/RM2005v060n02ABEH000823. 570, 572

[2] Buss, S. R., Bounded Arithmetic, vol. 3 of Studies in Proof Theory: Lecture Notes, Bibliopolis, Naples, Italy, 1986. Zbl 0649.03042. MR 0880863. 571, 574

[3] Feferman, S., "Arithmetization of metamathematics in a general setting," Fundamenta Mathematicae, vol. 49 (1960/61), pp. 35-92. Zbl 0095.24301. MR 0147397. 578

[4] Guaspari, D., and R. M. Solovay, "Rosser sentences," Annals of Mathematical Logic, vol. 16 (1979), pp. 81-99. Z Zbl 0426.03062. MR 0530432. DOI 10.1016/0003-4843(79)90017-2. 577

[5] Hájek, P., and P. Pudlák, Metamathematics of First-Order Arithmetic, Perspectives in Mathematical Logic, Springer, Berlin, 1993. Zbl 0889.03053. MR 1219738. DOI 10.1007/978-3-662-22156-3. 570, 571, 572

[6] Jeroslow, R. G., "Experimental logics and $\Delta_{2}^{0}$-theories," Journal of Philosophical Logic, vol. 4 (1975), pp. 253-67. Zbl 0319.02024. MR 0505406. 578

[7] Leivant, D., "The optimality of induction as an axiomatization of arithmetic," Journal of Symbolic Logic, vol. 48 (1983), pp. 182-84. Zbl 0515.03018. MR 0693260. DOI 10.2307/2273332. 572

[8] Lindström, P., Aspects of Incompleteness, 2nd ed., vol. 10 of Lecture Notes in Logic, Association for Symbolic Logic, Urbana, Ill., A K Peters, Natick, Mass., 2003. Zbl 1036.03002. MR 2014250. 578

[9] McAloon, K., "Formules de Rosser pour ZF," Comptes Rendus hebdomadaires des Séances de l'Académie des Sciences (Série A), vol. 281 (1975), pp. A669-72. Zbl 0333.02046. MR 0384543. 578 
[10] Montagna, F., "On the algebraization of a Feferman's predicate (the algebraization of theories which express Theor; X)," Studia Logica, vol. 37 (1978), pp. 221-36. Zbl 0398.03054. MR 0515168. DOI 10.1007/BF02124724. 578

[11] Montagna, F., and A. Sorbi, "Universal recursion-theoretic properties of r.e. preordered structures," Journal of Symbolic Logic, vol. 50 (1985), pp. 395-406. Zbl 0578.03026. MR 0793120. DOI 10.2307/2274228. 574

[12] Ono, H., "Reflection principles in fragments of Peano arithmetic," Zeitschrift für mathematische Logik und Grundlagen der Mathematik, vol. 33 (1987), pp. 317-33. Zbl 0607.03017. MR 0913295. DOI 10.1002/malq.19870330407. 571

[13] Orey, S., "Relative interpretations," Zeitschrift für mathematische Logik und Grundlagen der Mathematik, vol. 7 (1961), pp. 146-53. Zbl 0121.25501. MR 0146082. 579

[14] Pour-El, M. B., and S. Kripke, "Deduction-preserving 'recursive isomorphisms' between theories," Fundamenta Mathematicae, vol. 61 (1967), pp. 141-63. Zbl 0174.02003. MR 0252226. 573, 574

[15] Putnam, H., "Trial and error predicates and the solution to a problem of Mostowski," Journal of Symbolic Logic, vol. 30 (1965), pp. 49-57. Zbl 0193.30102. MR 0195725. 578

[16] Rosser, J. B., "Extensions of some theorems of Gödel and Church," Journal of Symbolic Logic, vol. 1 (1936), pp. 87-91. Zbl 0015.33802. 571, 577

[17] Selivanov, V., "Positive structures," pp. 321-50 in Computability and Models: Perspectives East and West, edited by S. B. Cooper and S. S. Goncharov, Kluwer Academic/Plenum, New York, 2003. MR 2043604. 574

[18] Shavrukov, V. Yu., "A smart child of Peano's," Notre Dame Journal of Formal Logic, vol. 35 (1994), pp. 161-85. Zbl 0815.03011. MR 1295557. DOI 10.1305/ndjfl/1094061859. 570, 578

[19] Smoryński, C., "Arithmetic analogues of McAloon's unique Rosser sentences," Archive for Mathematical Logic, vol. 28 (1989), pp. 1-21. Zbl 0663.03049. MR 0987763. DOI 10.1007/BF01624079. 570, 578

[20] Švejdar, V., "Modal analysis of generalized Rosser sentences," Journal of Symbolic Logic, vol. 48 (1983), pp. 986-99. Zbl 0543.03010. MR 0727788. DOI 10.2307/2273663. 580

[21] Tarski, A. A., Undecidable Theories, in collaboration with A. Mostowski and R. M. Robinson, Studies in Logic and the Foundations of Mathematics, North-Holland, Amsterdam, 1953. Zbl 0053.00401. MR 0058532. 569, 571, 578

[22] Verbrugge, R., and A. Visser, "A small reflection principle for bounded arithmetic," Journal of Symbolic Logic, vol. 59 (1994), pp. 785-812. Zbl 0814.03039. MR 1295969. DOI 10.2307/2275908. 580

[23] Visser, A., "Numerations, $\lambda$-calculus \& arithmetic," pp. 259-84 in To H. B. Curry: Essays on Combinatory Logic, Lambda Calculus and Formalism, edited by J. P. Seldin and J. R. Hindley, Academic Press, London, 1980. MR 0592807. 574

[24] Visser, A., "Peano's smart children: A provability logical study of systems with builtin consistency," Notre Dame Journal of Formal Logic, vol. 30 (1989), pp. 161-96. Zbl 0686.03033. MR 1050516. DOI 10.1305/ndjfl/1093635077. 578, 580

[25] Visser, A., "Can we make the second incompleteness theorem coordinate free?" Journal of Logic and Computation, vol. 21 (2011), pp. 543-60. Zbl 1262.03123. MR 2823429. DOI 10.1093/logcom/exp048. 575, 578

[26] Visser, A., "What is sequentiality?" pp. 229-69 in New Studies in Weak Arithmetics, edited by P. Cégielski, Ch. Cornaros and C. Dimitracopoulos, vol. 211 of CSLI Lecture Notes, CSLI Publications/Presses universitaires du Pôle de Recherche et d'Enseignement Supérieur de Paris-Est, Stanford/Paris, 2013. 570

[27] Visser, A., "The arithmetics of a theory," to appear in Notre Dame Journal of Formal Logic, preprint, 2012, http://dspace.library.uu.n1/handle/1874/234711. 570 
[28] Visser, A., "Peano Corto and Peano Basso: A study of local induction in the context of weak theories," Mathematical Logic Quarterly, vol. 60 (2014), pp. 92-117. DOI 10.1002/malq.201200102. 570

[29] Voorbraak, F., "A simplification of the completeness proofs for Guaspari and Solovay's R,” Notre Dame Journal of Formal Logic, vol. 31 (1989), pp. 44-63. Zbl 0712.03010. MR 1043791. DOI 10.1305/ndjfl/1093635332. 577

\section{Acknowledgments}

The question concerning the uniform density of Lindenbaum algebras was raised by Sy Friedman in conversation. Sy's question was inspired by the idea that perhaps the consistency operator is, in some sense, the least way to strengthen theories. This is not so, as is corroborated by the results of this paper. Both authors thank Lev Beklemishev for his consulting services. Visser's work was supported by the Templeton Foundation through his participation in the CRM Infinity Project (Templeton Foundation project \#13152).

Shavrukov

Nijenburg 24

1081GG Amsterdam

The Netherlands

v.yu.shavrukov@gmail.com

Visser

Department of Philosophy

Utrecht University

Janskerkhof 13A

3512BL Utrecht

The Netherlands

a.visser@uu.nl 